\title{
Metallographische Mitteilungen aus dem Institut für anorganische Chemie der Universität Göttingen. XXIV.
}

\section{Über die Legierungen des Nickels mit Antimon.}

\author{
Von \\ K. Lossew.
}

Mit 1 Figur im Text und 2 Tafeln.

Angaben über die Legierungen des Antimons mit dem Nickel fehlen fast vollständig. RössLER ${ }^{1}$ erwähnte beiläufig, dals beim Zusammenschmelzen von Nickel mit Antimon und darauf folgender Auflösung der Legierung kleine nadelförmige Kristalle zurückbleiben, die aus Nickel und Antimon bestehen. Wenn man diesen Hinweis mit der Tatsache des Vorkommens des Breithauptits NiSb in der Natur zusammenbringt, so kann man schon vermuten, dafs Nickel in geschmolzenem Antimon sich lösen wird. Vorläufige Versuche haben diese Vermutung bestätigt. Schon auf dem Gebläse bei etwa $1200^{\circ} \mathrm{kann}$ man leicht eine $50 \%$ ige Lösung erhalten.

Um die Zusammensetzung der Nickel-Antimon-Verbindungen zu bestimmen, suchte ich ein vollständiges Zustandsdiagramm auszuarbeiten.

Die Legierungen bis $80 \%$ Nickel erhielt man auf dem Gebläse im hessischen Tiegel, in welchem die Nickelstückchen mit Antimonstückchen überschüttet waren.

Die Legierungen mit 80 Gewichtsproz. Nickel, die zu nahe der Grenztemperatur des Gebläses vollkommen schmilzt, erhielt man im elektrischen Ofen und schmolz dann dieselbe von neuem auf dem Gebläse, so dafs für diese Konzentration die Abkühlungskurve unter denselben Bedingungen aufgenommen wurde wie auch für die Legierungen niederer Nickelkonzentrationen. Die Abkühlungskurven

${ }^{1}$ Rössler, Z. anorg. Chem. 9 (1895), 31; Chem. Centrbl. 1895 II, 179. 
der Legierungen von 90.5 Gewichtsproz. Nickel und für reines Nickel wurden erhalten, indem man im elektrischen Ofen arbeitete.

Als Ausgangsmaterial diente das reine Antimon Karmbadms und Nickel in Gestalt eines dicken Drahtes, der die Zusammensetzung hatte: ${ }^{1}$

$$
\begin{aligned}
& \mathrm{Ni} \text {. . } 97.9 \% \\
& \text { Co . . } 1.5 \% \\
& \mathrm{Fe} \cdot . \quad 0.6 \% \text {. }
\end{aligned}
$$

Zum Zwecke der Übertragung der erhaltenen Temperaturen auf die Skala des Luftthermometers wurden die von mir erhaltenen

\begin{tabular}{|c|c|c|c|}
\hline & Holbory und $D_{A Y}$ & von mir gefunden & Differenz \\
\hline non & . . $630.6^{\circ}$ & $620^{\circ}$ & $+10.6^{\circ}$ \\
\hline Gol & . . 1064 & 1053 & +11 \\
\hline Nickel & . . 1484 & 1435 & +49 \\
\hline
\end{tabular}
Daten für die Schmelztemperaturen des Antimons, des Goldes, des Nickels mit den Daten Horborss und Days verglichen.

Wie aus der Tabelle ersichtlich, bleibt die Differenz in den Temperaturgrenzen $620-1053^{\circ}$ konstant; für den Schmelzpunkt des Nickels $1435^{\circ}$ steigt sie stark an. Demgemäls nehmen wir diese Korrektion für Temperaturen von $620-1053^{\circ}$ als konstant an; für Temperaturen zwischen $1053^{\circ}$ und $1435^{\circ}$ aber als geradlinig wachsend mit der Temperatur von $11-49^{\circ}$.

In den Legierungen bis $33 \%$ Nickel trat eine ziemlich starke Verflüchtigung des Antimons ein, so dafs die Oberfläche der Legierung und die inneren Wände des Tiegels nach zwei aufeinander folgenden Schmelzungen sich mit einer zarten Kristallschicht von Antimonsäure bedeckten. Von 33 Gewichtsproz. Nickel an ist diese Schicht schon nicht mehr bemerkbar. Die folgende Tabelle gibt für je $30 \mathrm{~g}$ Schmelze die Gewichtsabnahme an Metall nach einer Schmelzung, woraus sich der Nickelgehalt nach der Schmelzung unter Annahme der Nichtflüchtigkeit des Nickels leicht berechnet:

(S. Tabelle, S. 60.)

Die Bestimmungen der Ablühlungskurven wurden für jede Legierung zweimal nacheinander an derselben Schmelze ausgefuhrt; wobei die Temperaturen der Haltepunkte für zwei Parallelversuche

1 Nach der Analyse, die im hiesigen Laboratorium von Dr. GokrtLER ausgeführt wurde. 


\begin{tabular}{c|c|c}
\hline \hline $\begin{array}{c}\text { Ursprüngliche Zusammensetzung } \\
\text { in Gewichtsproz. Nickel vor der } \\
\text { Schmelzung }\end{array}$ & $\begin{array}{c}\text { Gewichtsverlust } \\
\text { in } \mathbf{g}\end{array}$ & $\begin{array}{c}\text { Nickelgehalt } \\
\text { nach d. Schmelzung } \\
\text { in \% }\end{array}$ \\
\hline 7 & 2.62 & 7.67 \\
10 & 0.96 & 10.33 \\
16.6 & $1.93 ; 1.99$ & 17.83 \\
20 & 3.03 & 22.25 \\
25 & 3.28 & 28.07 \\
40 & 0.97 & 41.33 \\
50 & 0.25 & 50.42
\end{tabular}

stets dieselben waren, die Zeitdauer der Haltepunkte wichen für gröfsere Werte nicht mehr als 10 Sekunden voneinander $a b$, für kleine fielen sie gewöhnlich zusammen. In den Tabellen sind die arithmetischen Mittel von je zwei Beobachtungen aufgeführt. Die Temperaturen des Beginnes der Kristallisation zeigten infolge von Unterkühlung Differenzen von $10-15^{\circ}$. In den Tabellen werden im Falle von Unterkühlungen nicht die Mittelwerte, sondern nur die höchsten Temperaturen verzeichnet.

Die Analysen wurden nur für die ausgezeichneten Punkte der Schmelzkurve ausgeführt, und zwar für die Konzentrationen 25, 30, 47, 55 und 65 Gewichtsproz. Nickel. Bei den niedrigeren Konzentrationen des Nickels zeigt die Verflüchtigung des Antimons einen zu starken Einflufs auf die Zusammensetzung der Legierung, als dafs man ihn vernachlässigen könnte, und deshalb wurde an der Konzentration der Legierung eine durch geradlinige Interpolation ermittelte Korrektion angebracht. So verfuhr man für Legierungen von $25-47 \%$. Für Konzentrationen unter 25 Gewichtsproz. wurde auf Grupd der Bestimmungen der Gewichtsverluste des Regulus eine Korrektur vorgenommen. In den Tabellen sind die durch eine Analyse des Regulus ermittelten Konzentrationen mit einer einfachen Asteristik bezeichnet.

\section{(S. Tabelle 1-4, S. 61.)}

Für die Legierungen mit mehr als $55 \% \mathrm{Ni}$, wo eine Verflüchtigung fast nicht vorhanden ist, brauchten keine Korrekturen angebracht zu werden. In den Tabellen 1, 2, 3 sind beide Zusammensetzungen gegeben: in der ersten Kolumne ist die ursprüngliche Zusammensetzung von der Schmelzung, in der zweiten die Zusammensetzung nach zwei aufeinanderfolgenden Bestimmungen der Abkühlungskurven gegeben. 
Tabelle 1.

\begin{tabular}{|c|c|c|c|c|c|c|}
\hline \multicolumn{2}{|c|}{ Ni-Gewichtsprozent } & \multirow{3}{*}{$\begin{array}{l}\text { Beginn } \\
\text { der Kri- } \\
\text { stallisat. }\end{array}$} & \multirow{2}{*}{\multicolumn{2}{|c|}{$\begin{array}{c}\text { Erster } \\
\text { Haltepunkt }\end{array}$}} & \multirow{2}{*}{\multicolumn{2}{|c|}{$\begin{array}{c}\text { Zweiter } \\
\text { H\&ltepunkt }\end{array}$}} \\
\hline \multirow{2}{*}{$\begin{array}{c}\text { Ab- } \\
\text { gewogen }\end{array}$} & \multirow{2}{*}{$\begin{array}{l}\text { Korri- } \\
\text { giert }\end{array}$} & & & & & \\
\hline & & & Temperatur & Zeitdauer & Temperatur & Zeitdauer \\
\hline $0 \%$ & $0 \%$ & $631^{\circ}$ & - & - & Kristallisatio & nszeit 280" \\
\hline 1 & 1.1 & 624 & - & - & Unterkhl. 613 ${ }^{0}$ & $75^{\prime \prime}$ \\
\hline 2 & 2.2 & 622 & - & 一 & Geimpft 615 & 210 \\
\hline $\mathbf{3}$ & 3.8 & - & - & - & Onterkhl. 615 & 230 \\
\hline 4 & 4.3 & 698 & $612^{\circ}$ & $5^{\prime \prime}$ & \# 612 & 225 \\
\hline $\mathbf{5}$ & 5.4 & 722 & Unterkühl. 610 & 15 & 610 & 190 \\
\hline 6 & 6.6 & 774 & 613 & 15 & 613 & 190 \\
\hline 7 & 7.67 & 820 & 611 & 20 & 613 & 190 \\
\hline 10 & 11.0 & 896 & 610 & 30 & 607 & 155 \\
\hline 20 & 22.25 & 1058 & Unterkūhl. 616 & 45 & 608 & 90 \\
\hline 22.5 & 25.1 & 1075 & 613 & 50 & 608 & 80 \\
\hline 24.64 & 27.5 & 1096 & Unterkühl.616 & 55 & 611 & 60 \\
\hline 25 & $27.96^{*}$ & 1125 & \# 613 & $5 \dot{5}$ & 603 & 60 \\
\hline 27.5 & $\mathbf{3 0 . 3}$ & 1145 & 613 & 30 & , 593 & $\mathbf{3 0}$ \\
\hline 30 & $32.72^{*}$ & 1150 & - & - & Kristallisatior & nszeit $130^{\prime \prime}$ \\
\hline & & & Mittel $612^{\circ}$ & & $\overline{\text { Mittel } 611^{\circ}}$ & \\
\hline
\end{tabular}

Tabelle 2.

\begin{tabular}{c|c|c|c}
\hline \multicolumn{2}{c|}{$\begin{array}{c}\text { Ni-Gewichtsprozent } \\
\text { Abgewogen }\end{array}$} & $\begin{array}{c}\text { Beginn der } \\
\text { Kristallisation }\end{array}$ & $\begin{array}{c}\text { Ende der } \\
\text { Kristallisation }\end{array}$ \\
\hline 32.83 & 35.1 & $1158^{\circ}$ & - \\
35 & 37.1 & 1158 & $1143^{\circ}$ \\
37.5 & 39.3 & 1154 & 1120
\end{tabular}

Tabelle 3.

\begin{tabular}{|c|c|c|c|c|c|c|}
\hline \multicolumn{2}{|c|}{$\begin{array}{l}\text { Ni-Gewichts- } \\
\text { prozent }\end{array}$} & \multirow{2}{*}{$\begin{array}{c}\text { Beginn } \\
\text { der } \\
\text { Kristallisation }\end{array}$} & \multicolumn{2}{|c|}{$\begin{array}{c}\text { Erster } \\
\text { Haltepunkt }\end{array}$} & \multicolumn{2}{|c|}{$\begin{array}{l}\text { Zweiter Haltepunkt } \\
\text { der Umwandlung }\end{array}$} \\
\hline $\begin{array}{l}\text { Abge- } \\
\text { wogen }\end{array}$ & $\begin{array}{c}\text { Korri- } \\
\text { giert }\end{array}$ & & Temp. & $\begin{array}{l}\text { Zeit- } \\
\text { dauer }\end{array}$ & Teniperatur & $\begin{array}{l}\text { Zeit- } \\
\text { dauer }\end{array}$ \\
\hline $40 \%$ & $41.5 \%$ & $1148^{\circ}$ & - & - & $578^{\circ}$ & $15^{\prime \prime}$ \\
\hline 42.5 & 43.7 & 1124 & $1068^{\circ}$ & $25^{\prime \prime}$ & 581 & 30 \\
\hline 45 & 45.8 & 1102 & 1071 & 60 & 580 & 40 \\
\hline 47.0 & $47.60^{*}$ & - & 1072 & 120 & 576 & 50 \\
\hline 47.5 & 48.1 & 1090 & 1074 & 90 & 579 & 55 \\
\hline 50 & 50.4 & 1131 & 1076 & 45 & 582 & 70 \\
\hline 52.5 & 52.8 & Unterkühl. 1157 & 1071 & 20 & 583 & 75 \\
\hline \multirow[t]{2}{*}{55} & \multirow[t]{2}{*}{$55.12 *$} & \multirow[t]{2}{*}{$\Rightarrow \quad 1170$} & \multicolumn{2}{|c|}{ Krist.-Zeit 90" } & Intervall581-5710 & \multirow[t]{2}{*}{20} \\
\hline & & & Mitt.1072 & & Mittel $580^{\circ}$ & \\
\hline
\end{tabular}


Tabelle 4.

\begin{tabular}{|c|c|c|c|c|c|c|}
\hline \multirow[t]{2}{*}{$\begin{array}{c}\text { Ni- } \\
\text { Gewichts } \\
\text { prozent }\end{array}$} & \multirow[t]{2}{*}{$\begin{array}{c}\text { Beginn der } \\
\text { Kristalli- } \\
\text { sation }\end{array}$} & \multirow[t]{2}{*}{$\begin{array}{c}\text { Ende } \\
\text { der Kri- } \\
\text { stallisat. }\end{array}$} & \multicolumn{2}{|c|}{$\begin{array}{c}\text { Erster } \\
\text { Haltepunkt }\end{array}$} & \multicolumn{2}{|c|}{$\begin{array}{l}\text { Zweiter Haltepunkt } \\
\text { der Reaktion im } \\
\text { festem Zustande }\end{array}$} \\
\hline & & & Temperatur & Zeitd. & Temperatur & Zeitd. \\
\hline $57.5 \%$ & $1166^{\circ}$ & - & - & - & - & - \\
\hline 60 & Unterk. 1154 & - & $1096^{\circ}$ & $10^{\prime \prime}$ & $672^{\circ}$ & $\mathbf{2 5 \prime \prime}$ \\
\hline $65.21 *$ & - & - & 1103 & 95 & 673 & 50 \\
\hline 70 & 1179 & 一 & 1103 & 60 & 679 & 40 \\
\hline 80 & 1320 & 一 & 1102 & 35 & 680 & 25 \\
\hline 90 & 1393 & - & 1100 & 10 & 679 & $\mathbf{5}$ \\
\hline 95 & 1433 & $1409^{\circ}$ & - & - & - & 一 \\
\hline reines $\mathrm{Ni}$ & Unterk. 1484 & - & Kristallisation & 3zeit 80" & - & - \\
\hline & & & Mittel $1100^{\circ}$ & & Mittel $677^{\circ}$ & \\
\hline
\end{tabular}

Auf der Schmelzkurve hat man zwei deutlich ausgesprochene Temperaturmaxima $D$ und $F$, die den beiden Verbindungen: $\mathrm{NiSb}$ (32.83 Gewichtsproz. Nickel) und $\mathrm{Ni}_{5} \mathrm{Sb}_{2}$ (54.97 Gewichtsproz. Nickel) entsprechen.

$\mathrm{NiSb}$, das dem Minimum $D$ bei $1158^{\circ}$ entspricht, besitzt eine kupferrote Farbe und eine bedeutende Härte und Sprödigkeit. Während NiSb sich in Salpetersäure leicht löst, wirken die Säuren $\mathrm{HCl}, \mathrm{H}_{2} \mathrm{SO}_{4}$, wie auch die starken Basen nicht auf diese Verbindung ein.

Dafs wir es hier mit der Verbindung NiSb zu tun haben, ersieht man aus folgenden Tatsachen:

1. Die Abkühlungskurve der Legierung mit 32.72 Gewichtsproz. Nickel hat das für eine Verbindung charakteristische Aussehen und besitzt nur einen Haltepunkt.

2. Die Zeitdauer des Haltepunktes für den Übergangspunkt $C$ vermindert sich mit wachsendem Nickelgehalt und wird schliefslich bei $33 \% \mathrm{Ni}=0$.

3. Auf dem Schliffe der Legierung von 32.72 Gewichtsproz. Nickel sieht man nur eine Art von Kristallen, die durch feine Linien voneinander geschieden sind.

Die dem anderen Maximum $F$ bei $1170^{\circ}$ entsprechende Verbindung $\mathrm{Ni}_{8} \mathrm{Sb}_{2}$ ist noch härter, aber nicht so spröde wie die Verbindung NiSb, obwohl man auch sie verhältnismäfsig leicht pulverisieren kann; der Bruch ist feinkörnig von der Farbe des Gufseisens. 


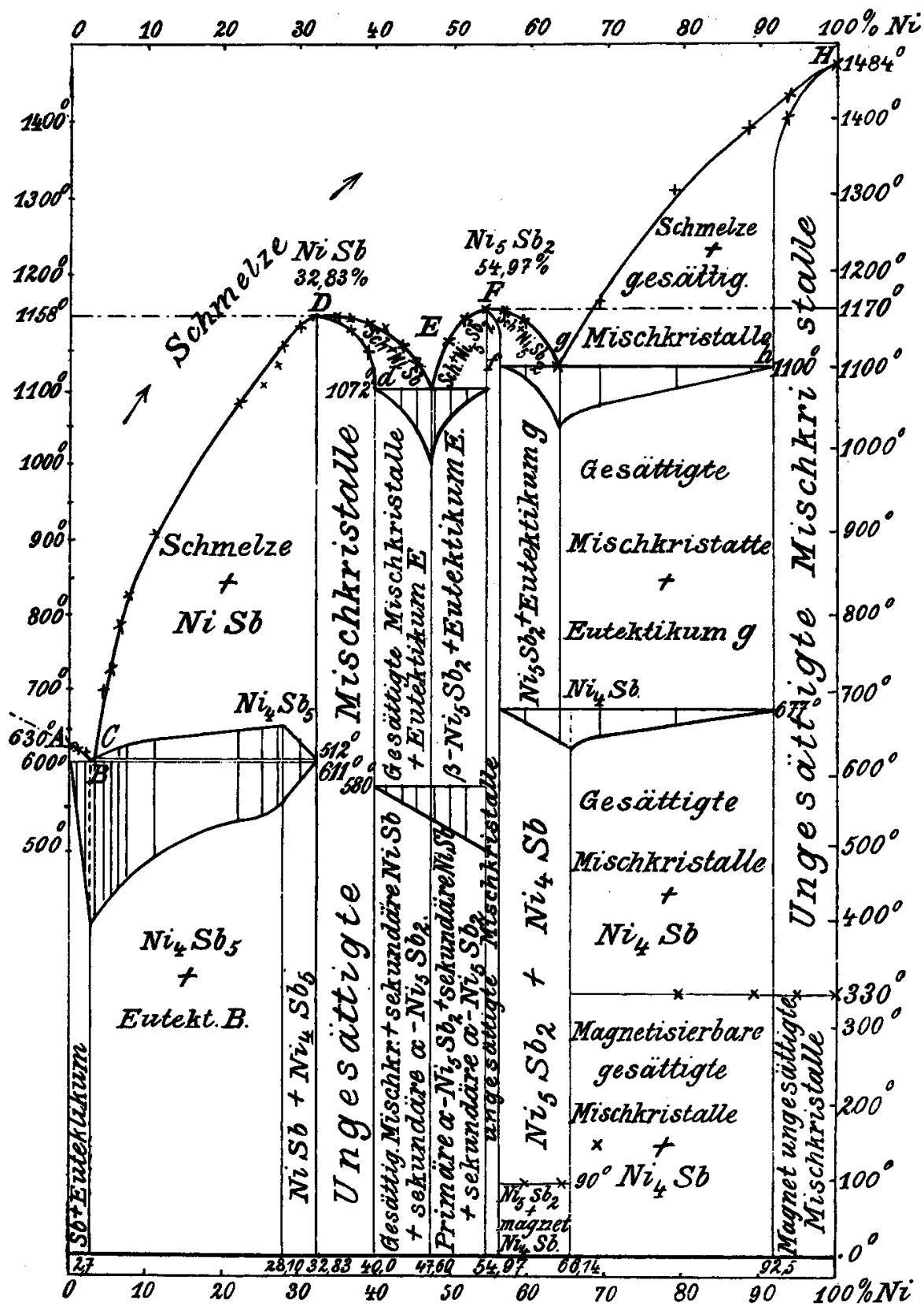

Fig. 1. 
Gegen Reagentien verhält sich die Verbindung $\mathrm{Ni}_{5} \mathrm{Sb}_{2}$ ebenso wie die vorhergehende, nur wird sie von Salpetersäure schwerer aufgelöst. Die Gründe, welche die Existenz dieser Verbindung beweisen, sind folgende:

1. Die Zeitdauer des eutektischen Haltepunktes bei $1072^{\circ}$ wird bei $55.0 \% \mathrm{Ni}$ gleich Null.

2. Die Zeitdauer der polymorphen Umwandlung bei $580^{\circ}$ nimmt von $40.0 \%$ bis 54.97 Gewichtsproz. Nickel beständig zu und erreicht für die Zusammensetzung $\mathrm{Ni}_{5} \mathrm{Sb}_{2}$ ihr Maximum.

3. Schliefslich ist auf dem Schliffe die Legierung mit 55.12\% $\mathrm{Ni}$ nur eine Art von Kristallen zu sehen, die durch dünne Linien voneinander getrennt sind.

Die polymorphe Umwandlung der Kristalle $\mathrm{Ni}_{6} \mathrm{Sb}_{2}$ wird auf den Abkühlungskurven durch einen deutlich ausgebildeten Haltepunkt kenntlich gemacht. Wenn der Nickelgehalt der ursprünglichen Schmelze denjenigen der Kristallart $\mathrm{Ni}_{5} \mathrm{Sb}_{2}$ etwas überschreitet, so wird hierdurch erstens die Zeitdauer der Umwandlung ganz aufserordentlich verkürzt und der Haltepunkt nimmt den Charakter eines Umwandlungsintervalles an. Die Legierung mit $55.12 \% \mathrm{Ni}$, welche nur $0.15 \% \mathrm{Ni}$ mehr enthält, als der Verbindung $\mathrm{Ni}_{5} \mathrm{Sb}_{2}$ entspricht, besitzt eine Umwandlungsdauer von 20 Sekunden, während dieselbe etwa 70 Sekunden betragen sollte. Bei den folgenden Legierungen von $55-66.1 \% \mathrm{Ni}$ ist von dem thermischen Effekt, welcher dieser Umwandlung entspricht, überhaupt nichts mehr wahrzunehmen. In diesem Falle liegen die Verhältnisse ganz ähnlich wie bei der Umwandlung der Kristallart $\mathrm{Zn}_{3} \mathrm{Sb}_{2}{ }^{1}$. Es scheint eine geringe Menge von Nickel, welche die Kristalle $\mathrm{Ni}_{5} \mathrm{Sb}_{2}$ gelöst enthalten, die Umwandlung zu verhindern oder sehr stark zu erniedrigen.

Die Streifung der Kristalle ist sowohl an den langsam, als auch an den schnell abgekühlten Schliffen wahrzunehmen. Man ersieht, dafs die Streifung auf den Schlifffächen der schnell abgekühlten Kristalle eine sehr regelmäfsige ist, während dieselbe auf den Schliffflächen der langsam abgekühlten Kristalle, wie anf Fig. 7, bedeutend weniger regelmälsig verläuft. Erwärmt man den langsam abgekühlten Schliff 7 Stunden lang auf $570-575^{\circ}$, so ist die Streifung auf einem Teil der Kristalle verschwunden.

1 Mönkzmryer, Z. anorg. Chem. 43, 192. 
Nehmen wir nun das Zustandsdiagramm der Antimon-Nickellegierungen genauer durch.

Von reinem Antimon an erniedrigt sich mit Vergröfserung der Nickelmenge die Temperatur des Beginnes der Kristallisation auf der Kurve $A B$ um etwa $19^{\circ}$. Bei der Ablühlung scheidet sich zuerst das Antimon in Gestalt teils abgerundeter, teils eckiger, bei schwachem Ätzen rosenfarbig anlaufender Kristalle aus, und darauf die körnige Masse des Eutektikums $B$, das die Kristallzwischenräume ausfullt, wie dies auf der Photographie des Schliffes in 1.1 Gewichtsproz. Nickel ersichtlich ist (Fig. 1).

Der eutektische Punkt $B$ selbst liegt bei der Temperatur $611^{\circ}$ zwischen 2 und 3 Gewichtsproz. Nickel. - Dies beweist der Umstand, dafs 1. das Zeitmaximum des eutektischen Haltepunktes auf $3 \%$ Nickel fallt, und 2. dafs die Strukturen der Schliffe mit $2 \%$ und $1 \% \mathrm{Ni}$ nicht wesentlich verschieden sind. - Bei $3 \% \mathrm{Ni}$ ist das primär ausgeschiedene Antimon nicht mehr vorhanden, dafür tritt auf dem gelben Fond des Eutektikums eine neue Art von primär ausgeschiedenen Kristallen in Form von weifsen, glänzenden Nadeln auf. Ein solches Aussehen der Kristalle ist der neuen Verbindung eigen, die sich unmittelbar aus der Schmelze auf dem Kurvenast $B C$ ausscheidet. Für dieselbe Verbindung, welche sich aber aus NiSb und der Schmelze bildet, ist die Dendritenform charakteristisch (Fig. 2).

Da die Zeitdauer der Kristallisation bei $612^{\circ}$ ihren maximalen Wert, welcher dem Punkte $B$ entspricht, bei 3.3\% Ni hat, so mufs auch der Übergangspunkt $C$ in unmittelbarer Nähe dieser Konzentration liegen.

Von 4 Gewichtsproz. Nickel an findet man auf den Abkühlungskurven zwei Haltepunkte. Das erste Halten der Temperatur tritt gewöhnlich ohne Unterkuhlung ein, während dem zweiten Halten immer eine Unterkühlung, wie sie für die Ausscheidung des Antimons charakteristisch ist, vorausgeht. Der Betrag dieser Unterkühlung erreicht zuweilen $80^{\circ}$. Unter dem Mikroskop sind bei stärkerer Vergröfserung auf allen Schliffen zwischen 3 und $32.83 \%$ drei Strukturelemente zu erkennen: eine gelbe Kristallart, deren Farbe bei wachsender Konzentration des Ätzmittels (Salpetersäure) in blau umschlägt, diese Kristallart ist mit einer weifsen Schicht umhüllt; in den Zwischenräumen befinden sich graue, unregelmäfsig geformte Massen von eutektischer Struktur. Die gelben, weifsumhüllten Kristalle treten, wie auch in Fig. $3 \mathrm{zu}$ erkennen ist, relief- 
artig über dem Eutektikum hervor, das Eutektikum ist also das weichste Strukturelement.

Diese Struktur der Legierungen weist darauf hin, dals die Kristalle von NiSb, welche sich auf der Kurve $C D$ abgeschieden haben, bei der Temperatur des Punktes $C$, bei $612^{\circ}$ mit der $3.3 \%$ Ni enthaltenden Schmelze unter Bildung einer neuen Verbindung, welche die gelb- oder blaugeätzten Kristalle NiSb als heller Saum umgibt (Fig. 3), reagieren. Diesen Umhüllungserscheinungen entsprechen bekannte Abnormitäten in den thermischen Erscheinungen, welche die Bestimmung der Formel der bei $612^{\circ}$ gebildeten Verbindung erschweren. ${ }^{1}$ In solchen Fällen wird die Zeitdauer der eutektischen Kristallisation nicht bei der Zusammensetzung der reinen Verbindung Null, sondern hat noch über diese hinaus merkliche Werte, wodurch wir eines wertvollen Hilfsmittels zur Feststellung der Formel der reinen Verbindung beraubt sind, infolgedessen ist auch aus der Lage des Maximums der Menge, welche bei $612^{\circ}$ reagieren, die $\mathrm{Zu}$. sammensetzung der Verbindung nicht mit derjenigen Sicherheit, wie in normalen Fällen abzuleiten. Das Maximum der Zeitdauer des Haltepunktes bei $612^{\circ}$ liegt bei der Zusammensetzung $27.96 \% \mathrm{Ni}$. Der Formel $\mathrm{Ni}_{4} \mathrm{Sb}_{5}$ würden $28.10 \% \mathrm{Ni}$ entsprechen.

Um diese Formel sicherer zu ermitteln, wurden folgende Versuche angestellt. Legierungen $\mathrm{z}$ wischen 25 und $30 \% \mathrm{Ni}$ wurden lange Zeit auf $612^{\circ}$ erhitzt, doch verschwand hierbei das Eutektikum nicht, offenbar ist die Umhüllung der Kristalle NiSb für Antimon impermeabel. Erhitzt man das zusammengeprefste Gemenge von pulverförmigem $\mathrm{Sb}$ mit pulverförmigem $\mathrm{Ni}$ (etwa $28 \% \mathrm{Ni}$ ), so beginnt bei etwa $600^{\circ}$ eine Reaktion, durch welche sich auch nach Entfernung des Bunsenbrenners die Temperatur auf $800^{\circ}$ bis $900^{\circ}$ hob. Pulverisierte man den Regulus mit $27.96 \% \mathrm{Ni}$, um die Hülle der NiSb.Kristalle zu zerbrechen, und erwärmte man das zusammengeprefste Pulver 8 Stunden lang auf $600-608^{\circ}$, so konnte man auf dem Schliffe des zusammengeprefsten und erhitzten Konglomerats das Eutektikum nicht mehr erkennen; das Konglomerat war aber sehr porös, so dafs sich wohl merkliche Mengen des Eutektikums der Auffindung entzogen haben können. Es schlugen also die Versuche zur Sicherung der Formel $\mathrm{Ni}_{4} \mathrm{Sb}_{5}$ fehl.

Die Verbindung NiSb vermag im kristallisierten Zustande einerseits kein Antimon aufzunehmen, was aus der Tatsache, dafs die

${ }^{2}$ G. Tammans, $Z$. anorg. Chem. 45 (1905), 24. 
Umwandlung bei $612^{\circ}$ und die eutektische Kristallisation bei $611^{\circ}$ bis zur Zusammensetzung, welche der Verbindung NiSb entspricht, sich verfolgen lassen, folgt; andererseits bildet die Verbindung NiSb eine Reihe von nickelreicheren Mischkristallen bis zum gesättigten Mischkristalle $d$ mit $40.0 \%$ Ni bei $1072^{\circ}$.

Die Existenz dieser Reihe von Mischkristallen folgt sowohl aus der Form der Abkühlungskurven, auf denen sich deutliche Kristallisationsintervalle finden, als auch aus der Struktur der Kristallkonglomerate; dieselben bestehen aus einer einzigen Kristallart, deren einzelne Individuen voneinander durch feine Linien getrennt sind. Diese Linien haben auf der Schliffläche des Regulus mit $41.5 \% \mathrm{Ni}$ (Fig. 4) schon deutlich an Dicke zugenommen, offenbar weil hier schon etwas Eutektikum vorhanden ist. Auf den Schlifflächen des gesättigten Mischkristalles (Fig.4) sieht man parallel geordnete Streifen, deren Deutlichkeit mit wachsendem Sb-Gehalt abnimmt.

Bei weiterer Vergröfserung der Konzentration des Nickels wächst die Zeitdauer des eutektischen Haltepunktes; man sieht, dals auf den Schlifffä̈chen die Menge des sekundär ausgeschiedenen Eutektikums zunimmt. Bei $47.60 \% \mathrm{Ni}$ erreicht die Zeitdauer des eutektischen Haltepunktes ihr Maximum. Die Abkühlungskurve für diese Legierung hat keinen. Knick, sondern nur einen Haltepunkt. Unter dem Mikroskop sieht man nur ein Strukturelement - ein Eutektikum von selten schöner lamellarer Struktur (Fig. 5).

Auf dem Aste $E F$ wächst die Temperatur des Beginnes der Kristallisation, und die Zeitdauer des eutektischen Haltepunktes bei $1072^{\circ}$ nimmt von $47.60 \%$ bis zu $54.97 \% \mathrm{Ni}$ ab. Auf dem Fond des Eutektikums erscheint eine immer gröfser werdende Menge der primär ausgeschiedenen Verbindung $\mathrm{Ni}_{5} \mathrm{Sb}_{2}$ in Form abgerundeter Kristalle, die mit braunen Streifen bedeckt sind (Fig. 6). Bei steigendem Nickelgehalt scheint sich in den Kristallen der Verbindung $\mathrm{Ni}_{5} \mathrm{Sb}_{2}$ etwas Nickel zu lösen. Hierfür sprechen folgende Umstände: erstens, dafs die Zeitdauer der eutektischen Kristallisation bei $1100^{\circ}$ schon bei $57.0 \% \mathrm{Ni}$ anstatt bei $55.0 \% \mathrm{Ni}$ Null wird, und zweitens dafs der Haltepunkt bei $580^{\circ}$, wenn der Nickelgehalt den der Verbindung $\mathrm{Ni}_{6} \mathrm{Sb}_{2}$ auch nur wenig überschreitet, sich in ein undeutliches Umwandlungsintervall verwandelt. Die Konzentration des gesättigten Mischkristalles $f$ mufs bei etwa $57.0 \%$ Ni liegen, da im Schliff mit 57.5\% Ni schon eine kleine Menge des Eutektikum $G$ zu erkennen ist. Mit steigendem Nickelgehalt gelangt man dann zum eutektischen Punkte $G$, von dem aus die Temperatur des Be- 
ginnes der Kristallisation bis zum Schmelzpunkt des Nickels $H$ steigt. Auf dem Aste der Schmelzkurve $F G$ kristallisieren Mischkristalle, deren Zusammensetzung durch die Linie $F f$ gegeben ist. Auch auf dem Aste $G H$ scheiden sich Mischkristalle aus, deren Konzentration den Punkten der Kurve $H h$ entspricht. Die Gestalt dieser Kurve ist etwas ungewöhnlich; dieselbe wurde auf Grund der Lage des Punktes $h$, des Endes der Kristallisation der Schmelze, mit 95\% Ni und des Nickelschmelzpunktes angenommen. Die Konzentration des gesättigten Mischkristalles $h$ ergibt sich aus der Abhängigkeit der Zeitdauer der eutektischen Kristallisation von der Konzentration zu 92.5\% Ni. Dieses Resultat wird durch die Struktur der Legierungen bestätigt, denn der Schliff mit $90 \%$ läfst noch ein wenig Eutektikum, von dem ein Teil auf Fig. 12 schwarz erscheint, der andere aber eine später zu besprechende Umwandlung erlitten hat, erkennen, dagegen besteht die Legierung mit $95 \% \mathrm{Ni}$ aus unter sich homogenen Kristallen, welche durch feine Linien voneinander getrennt sind.

Bei $677^{\circ}$ tritt in den Legierungen von $57-92.5 \% \mathrm{Ni}$ ohne Unterkühlung eine Reaktion ein, welche auf den Abkühlungskurven einen deutlichen Haltepunkt hervorruft, die Zeitdaner desselben hat bei $66.0 \% \mathrm{Ni}$ ein Maximum. Die mikroskopische Beobachtung lehrt, dafs sich bei jener Temperatur aus zwei verschiedenen Kristallarten eine neue gebildet hat, welche - wie wir auf Fig. 8 sehen die primär ausgeschiedenen Kristalle des gesättigten Mischkristalles $f$ umgibt, und diese von dem dunkeln Eutektikum trennt. Man kann sich bei der Betrachtung von Fig. 8 wohl kaum des Eindrucks erwehren, dafs sich die Kristallart, welche die primär ausgeschiedenen gestreiften Kristalle umgibt, sich aus diesen und dem Eutektikum $G$ gebildet hat. Diese erst später bei $677^{\circ}$ gebildete Kristallart erkennt man auf Fig. 10 in einer Menge direkt aneinandergrenzender Polygone wieder. Da zwischen. denselben sich kein Eutektikum befindet, der Regulus also fast nur ans der sich bei $677^{\circ}$ bildenden Verbindung besteht, so kann die Zusammensetzung derselben nur wenig vom Gehalt $65.21 \%$ abweichen. Das Maximum der Zeitdauer der Reaktion bei $677^{\circ}$ liegt in Übereinstimmung hiermit bei $66.0 \%$ Ni. Der Formel $\mathrm{Ni}_{4} \mathrm{Sb}$ würde ein Nickelgehalt von $66.14 \% \mathrm{Ni}$ entsprechen. $\mathrm{Da}$ die Zeitdauer der Haltepunkte bei $677^{\circ}$ einerseits bei $57.0 \% \mathrm{Ni}$ und andererseits bei $92.5 \% \mathrm{Ni} \mathrm{Null}$ wird, so hat sich die Verbindung $\mathrm{Ni}_{4} \mathrm{Sb}$ bei $677^{\circ}$ aus den beiden gesättigten Mischkristallen $f$ und $h$, mit $57.0 \%$ und $92.5 \% \mathrm{Ni}$, nach folgender Gleichung gebildet: 


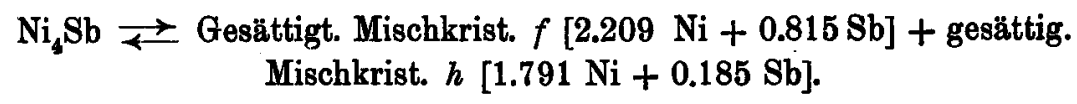

Diese Reaktion ist reversibel, denn erhitzt man den langsam gekuhlten Regulus mit $65.2 \% \mathrm{Ni}$, Fig. 10, der fast nur aus der Verbindung $\mathrm{Ni}_{4} \mathrm{Sb}$ besteht, kurze Zeit auf $700^{\circ}$ und schreckt dann denselben in Wasser ab, so ändert sich seine Struktur vollständig. Die Schlifflächen des abgeschreckten Regulus gibt Fig. 9 wieder. Man sieht hier ein faseriges Gefüge; die Legierung besteht offenbar aus zwei Strukturelementen, den gesättigten Mischkristallen $f$ und $h$. Aufserdem finden sich auf den Abkühlungskurven der bis $700^{\circ}$ wieder erhitzten Reguli dieselben Haltepunkte, welche man das erste Mal bei der Abkühlung der Schmelze fand, wieder. Beim Erhitzen vollzieht sich also die Reaktion nach der obigen Gleichung von links nach rechts und bei der Abkühlung von rechts nach links. Durch Abschrecken in Wasser kann die Reaktion ubersprungen werden und der Zustand der Legierungen über $677^{\circ}$ fixiert werden. Läfst man die Legierung mit $65.2 \% \mathrm{Ni}$ frei an der Luft sich abkühlen, so sieht man auf der Schlifffläche eines solchen Regulus, dafs die Bildung der Verbindung $\mathrm{Ni}_{4} \mathrm{Sb}$ nur in einigen Punkten eingetreten ist, während die Grundmasse noch aus dem Eutektikum $a$, dem Gemenge der beiden gesättigten Mischkristalle $f$ und $h$ besteht. Die im Tiegel langsam abgekühlten Legierungen mit $57-66.1 \% \mathrm{Ni}$ enthalten aufser dem gesättigten Mischkristall $f$, die denselben umhüllende Verbindung $\mathrm{Ni}_{4} \mathrm{Sb}$ und das Eutektikum $G$ mit einem kleinen Überschufs des gesättigten Mischkristalles $f$, da die Verbindung $\mathrm{Ni}_{4} \mathrm{Sb}$ etwas nickelreicher als das Eutektikum $G$ ist. Die ebenso gekthlten Legierungen mit 66.1-92.5\% Ni enthalten aber den unveränderten, primär abgeschiedenen Mischkristall $h$, welcher von der Verbindung $\mathrm{Ni}_{4} \mathrm{Sb}$ und dann noch einem kleinen Rest des Eutektikums $G$ umgeben ist. In Fig. 11 sind die hellen Teile der gesättigte Mischkristall $h$, dessen Konturen durch dunkle Schatten verbreitert sind, weil der Mischkristall $h$ als weicheres Strukturelement tief ausgeschliffen ist. Die weifsen, dunkel umrandeten Mischkristalle sind von der Verbindung $\mathrm{Ni}_{4} \mathrm{Sb}$, zwischen der sich wenig dunkelgefärbtes Eutektikum befindet, umgeben. Auf der Schlifffläche der Legierung mit $90 \% \mathrm{Ni}$, Fig, 12, hat die Menge des Mischkristalles $h$ bedeutend zugenommen, während die Menge der Verbindung $\mathrm{Ni}_{4} \mathrm{Sb}$ stark abgenommen und die des Eutektikums sich nicht wesentlich geändert hat. Bei langsamerer Kühlung nimmt die 
Menge des Eutektikums ab, indem sich die Menge $\mathrm{Ni}_{4} \mathrm{Sb}$ vermehrt. Offenbar tritt durch die Verbindung $\mathrm{Ni}_{4} \mathrm{Sb}$ hindurch ein Stoffaustausch zwischen dem Eutektikum und dem Mischkristall $h$ ein.

Die Legierungen von 100-57\% Ni sind bei Zimmertemperatur magnetisierbar. Die magnetische Permeabilität nimmt mit abnehmendem Nickelgehalt bis $70 \%$ stark ab, bei $57 \% \mathrm{Ni}$ ist dieselbe fast verschwunden, und die Legierungen, welche weniger als $55 \% \mathrm{Ni}$ enthalten, wirken auf eine empfindliche Magnetnadel nicht mehr ein. Die Temperaturen, bei denen die Magnetisierbarkeit der magnetisch permeabeln Legierungen fast vollständig verschwindet, wurden mit Hilfe einer ziemlich empfindlichen Magnetnadel bestimmt, es ergaben sich folgende Temperaturen:

\begin{tabular}{c|c|c}
\hline Ni-Gewichtsproz. & $\begin{array}{c}\text { Temp. des Verschwindens } \\
\text { der Magnetisierbarkeit }\end{array}$ & $\begin{array}{c}\text { Temp. der Wiederkehr } \\
\text { der Magnetisierbarkeit }\end{array}$ \\
\hline 100 & 330 & 325 \\
95 & 330 & 325 \\
90 & 320 & 310 \\
80 & 320 & 310 \\
70 & 160 & 150 \\
65 & 90 & 80 \\
60 & 90 & 80
\end{tabular}

Die Temperatur des starken Abfalles der Magnetisierbarkeit ändert sich bei den nickelreichen Legierungen innerhalb der Versuchsfehler nicht merklich. Die Verbindung $\mathrm{Ni}_{4} \mathrm{Sb}$ verliert bei $90^{\circ}$ ihre geringe Magnetisierbarkeit. Man könnte daher erwarten, dafs die Legierungen von $66-92 \% \mathrm{Ni}$, da dieselben alle aus dem Mischkristall $h$ und der Verbindung $\mathrm{Ni}_{4} \mathrm{Sb}$ bestehen, den grölsten Teil ihrer magnetischen Permeabilität bei $330^{\circ}$ und den geringen Teil derselben, der von der Gegenwart der Verbindung $\mathrm{Ni}_{4} \mathrm{Sb}$ herrührt, bei $90^{\circ}$ verlieren werden. $\mathrm{Da}$ aber nur eine qualitative Methode zur Anwendung kam, so liefs sich natürlich diese Forderung nicht erweisen.

Nickel bildet mit Antimon vier Verbindungen, nämlich: NiSb, $\mathrm{Ni}_{5} \mathrm{Sb}_{2}, \mathrm{Ni}_{4} \mathrm{Sb}_{5}$ (?) und $\mathrm{Ni}_{4} \mathrm{Sb}$. In den beiden ersten Verbindungen treten Ni und Sb mit Valenzen, die ihnen auch in ihren Sauerstoffverbindungen zukommen auf. In der Verbindung NiSb könnte sowohl $\mathrm{Ni}$ als auch $\mathrm{Sb}$ dreiwertig sein, in der Verbindung $\mathrm{Ni}_{5} \mathrm{Sb}_{2}$ wirde das Nickel zweiwertig und das Antimon fünfwertig sein. Diese Verbindungen schmelzen zu homogenen Flüssigkeiten, während die 
Verbindung $\mathrm{Ni}_{4} \mathrm{Sb}_{5}$ beim Schmelzen in eine Flüssigkeit und die Verbindung $\mathrm{NiSb}$ zerfällt. Die Verbindung $\mathrm{Ni}_{5} \mathrm{Sb}_{2}$ besitzt bei $580^{\circ}$ einen Umwandlungspunkt.

Besonders interessant ist die Verbindung $\mathrm{Ni}_{4} \mathrm{Sb}$, die nur unterhalb $677^{\circ}$ existiert und oberhalb dieser Temperatur in zwei gesättigte Mischkristalle mit 57 und $92.5 \%$ Ni zerfällt.

Zum Schlusse erachte ich es für meine Pflicht, Herrn Prof. G. Tammann für die Anregung zu dieser Arbeit meinen besten Dank auszusprechen.

Göttingen, Institut für anorganische Chemie der Universität.

Bei der Redaktion eingegangen am 16. Februar 1906. 


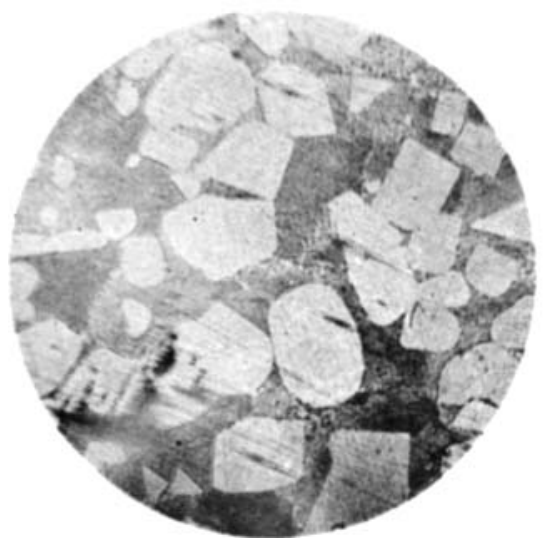

Fig. 1. $1,1 \% \mathrm{Ni},+98,9 \% \mathrm{Sb}$. 65 fache Vergrößerung. Geätzt mit $\mathrm{HNO}_{3}$.

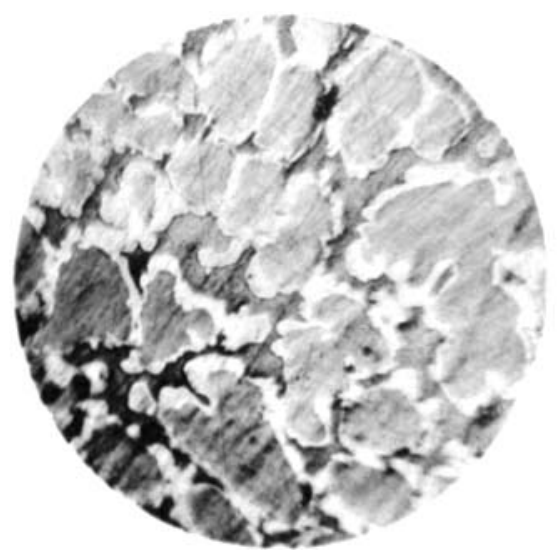

Fig. 3. $22,25 \%$ Ni. $+77,75 \%$ Sb. 190 fache Vergrößjerung.
Geätzt mit $\mathrm{HNO}_{3}$.

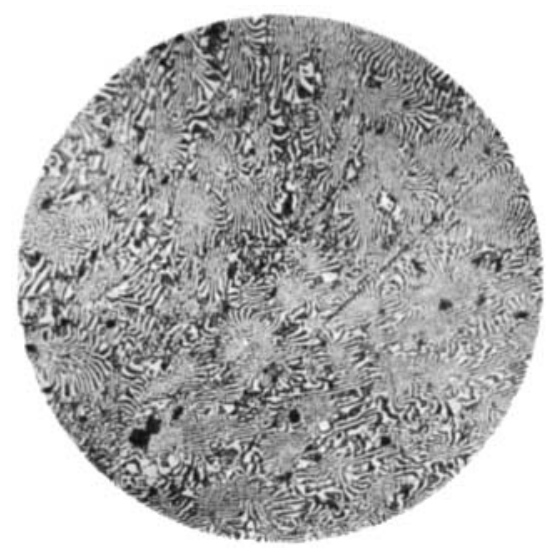

Fig. 5. $47,60 \%$ Ni. $+52,40 \%$ Sb. 65 fache Vergrößerung. Geätzt mit $\mathrm{HNO}_{\mathfrak{s}}$.

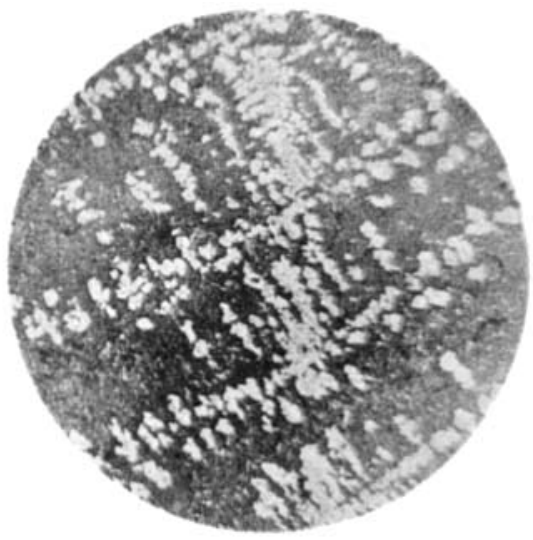

Fig. 2. $5,4 \% \mathrm{Ni}+94,6 \% \mathrm{Sb}$. 65 fache Vergrößerung. Geätzt mit $\mathrm{HNO}_{3:}$.

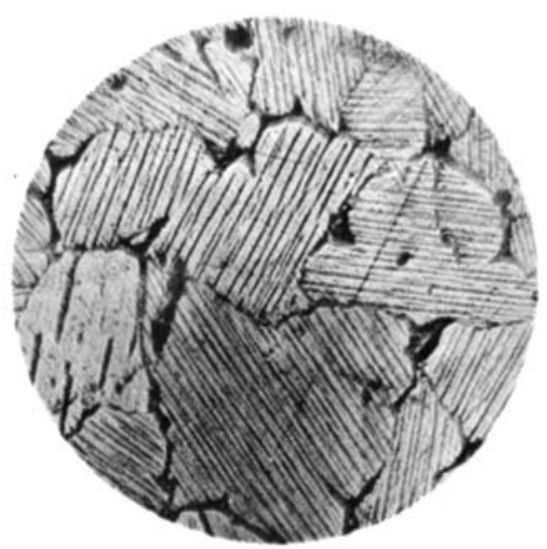

Fig. 4. $41,5 \% \mathrm{Ni} .+58,5 \%$ Sb. $6 \bar{f}$ ache Vergrößerung. Geätzt mit $\mathrm{HNO}_{3}$.

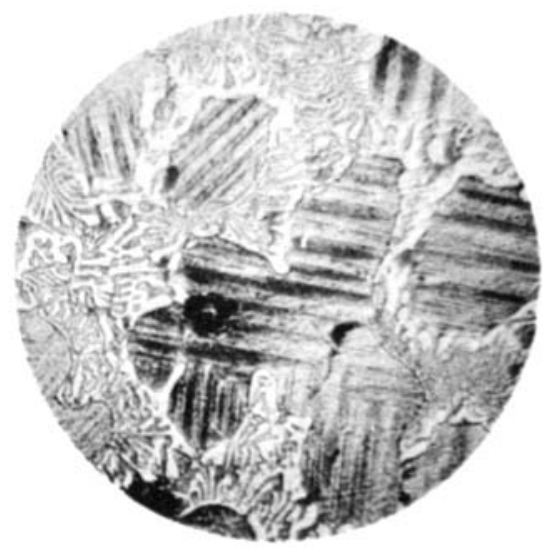

Fig. 6. $50,4 \%$ Ni. $+49,6 \%$ Sb. 190 fache Vergrößerung. Geätzt nit $\mathrm{HNO}_{3}$. 


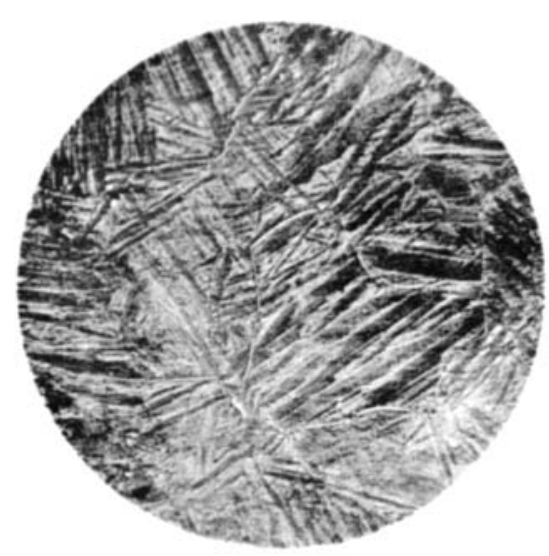

Fig. 7. $55,12^{\circ} \% \mathrm{Ni},+44,88^{\circ} \% \mathrm{Sb}$.

Bei gewöhnlichen Bedingungen abgekühlt. 65 fache Vergrößerung.
Geätzt mit HNO

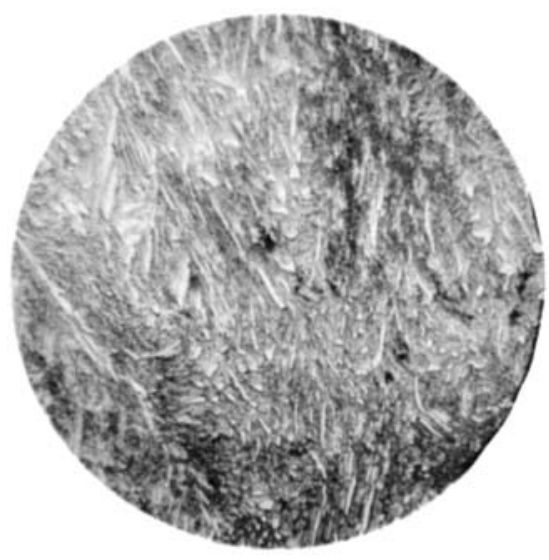

Fig. 9. $65,21 \%$ Ni. $+34,79 \%$ Sb. Abgeschreckt. 190 fache Vergrößlerung. Geätzt mit $\mathrm{HNO}_{3}$.

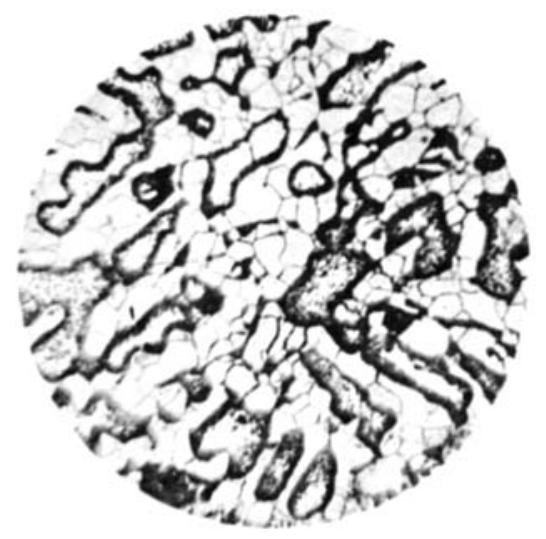

Fig. 11. $70 \%$ Ni. $+30 \%$ Sb. 190 fache Vergrößerung. Geätzt mit $\mathrm{HNO}_{\mathbf{j}}$.

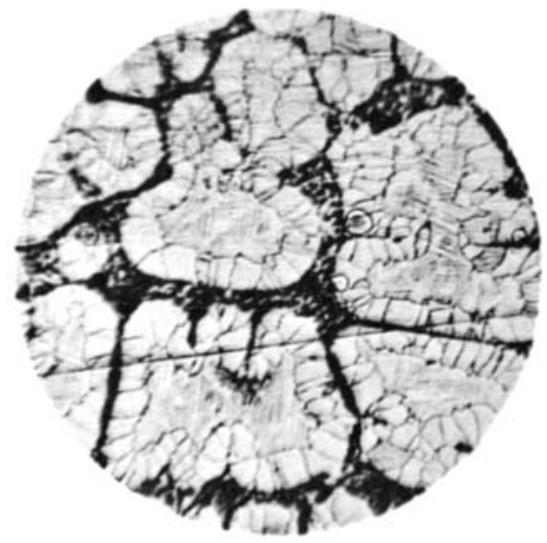

Fig. 8. $60 \% \mathrm{Ni} .+40 \%$ Sb. 145 fache Vergrößerung. Geätzt mit $\mathrm{HNO}_{3}$.

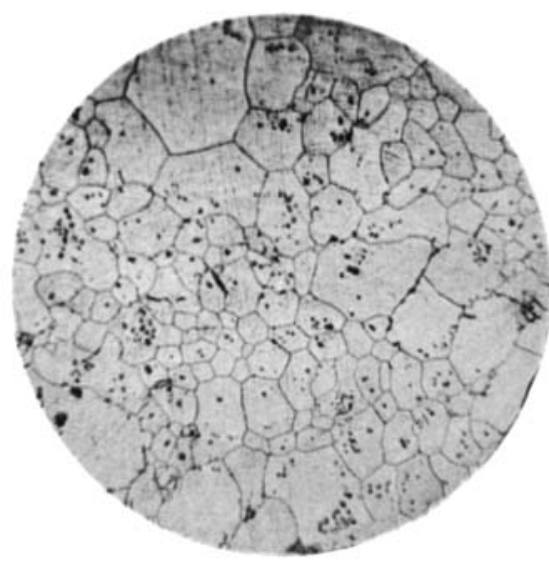

Fig. 10. 65,21\% Ni. $+34,79 \%$ Sb. Sehr langsam abgekühlt. 190 fache Vergrößerung. Geätzt mit $\mathrm{HNO}_{3}$.

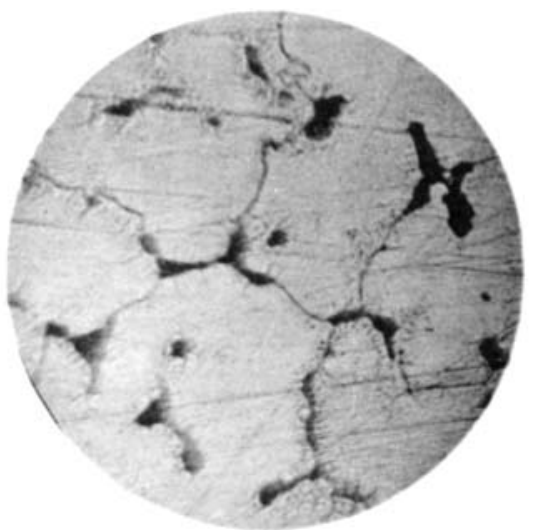

Fig. 12. $90 \% \mathrm{Ni} .+10 \% \mathrm{Sb}$. 190 fache Vergröbjerung. Geätzt mit $\mathrm{HNO}_{3}$. 